\title{
Vector Map Constrained Path Bundling in 3D Environments
}

\author{
Matthias Thöny \\ Department of Informatics \\ University of Zürich \\ mthoeny@ifi.uzh.ch
}

\author{
Renato Pajarola \\ Department of Informatics \\ University of Zürich \\ pajarola@ifi.uzh.ch
}

\begin{abstract}
Dense line graphs and polyline maps are challenging for interactive visualization in geographic information systems (GIS). Bundling techniques are a common approach to reduce clutter and have successfully been demonstrated for the display of complex planar graphs. Previous techniques typically employed some form of attraction or repulsion forces to bundle edges in two dimensions, and while in principle extensible to 3D they do not directly support hard intersection constraints in a 3D environment. In geographic visualization systems, e.g. such as interactive virtual globes or 3D GIS viewers, it is often necessary to take the $3 \mathrm{D}$ environment into account and to: (1) bundle lines and paths in 3D, (2) constrain path bundles to follow some reference network vector map, as well as (3) avoid intersections with the digital elevation model (DEM). In this paper we introduce a novel method which uses geographic vector map reference information to route, visualize and simplify path bundles along their network paths in a constrained 3D environment using adaptive B-splines. Moreover, we describe an efficient rendering architecture to flexibly display bundled paths within a 3D rendering pipeline at varying level of detail (LOD).
\end{abstract}

\section{Categories and Subject Descriptors}

I.3.5 [Computer Graphics]: Computational Geometry and Object Modeling

\section{General Terms \\ Algorithms}

\section{Keywords}

Geographic information visualization, vector maps, graph bundling, interactive rendering, large scale visualization

\section{INTRODUCTION}

An interesting challenge in geographic visualization is the analysis of transportation relations. These relations are represented by dense graphs containing many point-to-point connections. The edges represent semantic information, such a commuter connection between

Permission to make digital or hard copies of part or all of this work for personal or classroom use is granted without fee provided that copies are not made or distributed for profit or commercial advantage and that copies bear this notice and the full citation on the first page. Copyrights for thirdparty components of this work must be honored. For all other uses, contact the Owner/Author. Copyright is held by the owner/author(s).

IWGS'15, November 03-06, 2015, Bellevue, WA, USA

ACM 978-1-4503-3971-1/15/11.

http://dx.doi.org/10.1145/2833165.2833168 towns or an airline connection between two airports. The geographical location for this traffic relations allows the visual identification of traffic highlights, such as traffic jams or very frequently visited nodes. These traffic relation graphs can become large graphs with several thousands of connections and suffer from cluttering effects.

Effective visualizations of large scale and dense graphs or networks commonly use so called graph bundling techniques. The goals of graph bundling are first the reduction of visual clutter in a dense graph dataset, and second the highlighting of major connectivity paths and patterns (see e.g. Fig. 8) within this dataset. Individual as well as groups of related connections in a dense graph are very hard to identify. Bundling of paths leads to a visualization which makes it easier to analyze the prevalent connectivity structures.

Bundling dense point-to-point graphs, such as e.g. commuter data, may have to be constrained to and visualized over a variety of complex vector map reference networks, such as e.g. roads or train routes. This additional vector map often is the bases for some routing constraints that map the dense point-to-point connections to paths over the reference network. A visualization of such paths following the reference network constraints enriches the effective information visualization and visual analysis task. However, the density of the paths and complexity of the underlying reference network may cause visual cluttering and loss of the larger context of connectivity information.

Reducing clutter and highlighting major structural link information is even more important and challenging in an interactive geovisualization when using a $3 \mathrm{D}$ environment. In this paper we assume paths and graphs having nodes and edges with 3D coordinates within a geospatial framework including 3D terrain information and 3D obstacles. In 3D, it becomes harder to analyze, identify and bundle path trajectories in a dense graph constrained to a reference network due to the added interference, i.e. intersections and occlusions from 3D objects and obstacles.

In this work we present a novel vector map constrained path bundling method, that takes a dense graph over spatial locations and generates a variable level-of-detail bundling constrained to a reference vector map while avoiding intersections with given obstacles in 3D. See also overview shown in Fig. 1. In particular, we demonstrate our approach based on dense geo-spatial point-to-point relations that are routed and bundled over geographical vector maps, all embedded and visualized in a 3D digital terrain elevation model. Furthermore we are going to compare our results with state-of-the art techniques to the extent to which they may be applicable. 


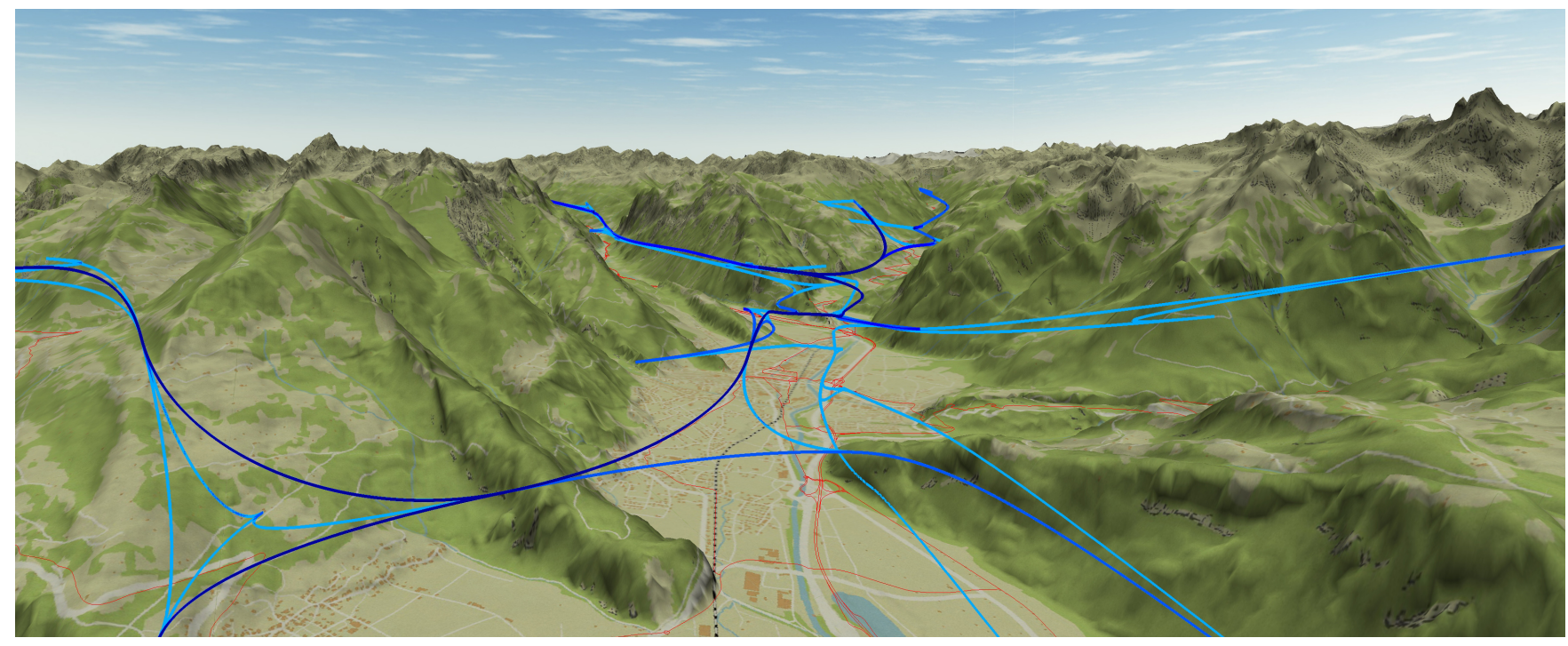

Figure 1: Example of a perspective view showing bundled paths over the 3D terrain of the Vorarlberg dataset.

\section{RELATED WORK}

A general overview of clutter reduction in graphs and information visualization is discussed e.g. in [4] and taxonomies for graph visualizations are described in [17].

Clutter reduction techniques can be classified as edge or node based problems. Of these, the former applies to this work as the georeferenced start- or end-points are intentionally left unmodified. Edge bundling methods can further be subdivided into two categories, geometry or image based methods. Geometry based methods use and manipulate the graph edges on the geometric object level, whereas image based methods solve the problems primarily in discretized image space and see the problem in a 2D planar projection. Our method belongs to the category of geometry based edge bundlings. However, in contrast to other methods, our aim is a 3D constrained geometric bundling suitable for interactive visualization in a 3D environment including correct visibility and depth occlusions in perspective 3D views. Recently published geometric graph bundling methods include force-directed edge bundling (FDEB) [10], divided-edge bundling [19], multilevel agglomerative edge bundling (MINGLE) [6], winding roads [16], geometrybased edge clustering for graph visualization [2] and hierarchical edge bundles [9].

FDEB [10] applies a field force to subdivided segments of a graph, such that subdivision points can be moved according to their attracting force directions. The algorithm has a high algorithmic complexity, but also avoids any complex hierarchical data structures. An extension of FDEB using different optimization constraints is described in [19]. The idea of force-directed edge bundling could potentially be extended to $3 \mathrm{D}$. The main challenge, however, is the identification of edge compatibility measurements which are working for all 3D line situations when using a graph dataset with 3D nodes and edges.

The MINGLE algorithm [6] is also based on the idea of optimization similar to FDEB. In MINGLE, the optimization is done with respect to limiting pixel overdraw, also called ink minimization, according to neighborhood information extracted from a 4D line interpretation. The method is proven to be faster than FDEB, how- ever, the ink saving algorithm assumes that all edges merge at predefined control points, which can produce esthetically insufficient results.

Another approach for edge bundling is the usage of control hierarchies. Geometry-based graph bundling [2] uses a control mesh forcing edges to pass through control points. The edge data is used to extract a control mesh and find a 2D partitioning layout, but the artificial origin of this control mesh does not imply any semantic meaning to the final result. Geometry-based graph bundling could possibly be extended to $3 \mathrm{D}$ datasets using a more complex control mesh and smoothing algorithm, but it is not clear how. Hierarchical edge bundles described in [9] are using hierarchical tree or graph structures. This approach is well known to visualize nodes in a hierarchical correlation. The application of hierarchical edge bundles to a $3 \mathrm{D}$ visualization can be found in [1]. However, visualizing hierarchical graph or tree structures is a different problem setup from normal graphs or applying a constraint network and thus not directly applicable or comparable.

Similar to the previous approaches, the Winding Roads algorithm described in [16] uses an artificial grid for routing bundles. The algorithm shows examples with a Voronoi grid structure and a quadtree grid structure. The edge routing is done based on a shortest path search. In our work we adapt and extend the idea of this algorithm in a more general way. We will show how the idea of edge routing can be applied to general reference graphs and we will extend this approach with a 3D line level-of-detail (LOD) method. There is another extension to the Winding Road algorithm showing one possible way of a 3D impression [15]. This extension uses a 2D approach and the final results are done using bump mapping for the 3D impression. However, bump mapping is a $2 \mathrm{D}$ effect for a 3D impression and it will not be possible to visually separate bundles in 3D environments if needed for example for skew lines. The bump mapping effect can be applied on almost any method, including image based methods. An example is shown in [12]. Recent and important image based methods are: graph bundling by kernel density estimation (KDEEB) [12] or skeleton based edge bundling (SBEB) [5]. 
$\operatorname{KDEEB}[12,13,11]$ is based on the idea of using the spatial edge density for clustering of graph edges, streaming graphs or graph sequences. This can be done very fast and does not require any special data structures. The method also shows some results for obstacle avoidance. SBEB [5] use distance fields to iteratively attract edges against a skeleton interpretation of the graph. These distance fields are generated by a 2D clustering of the graph edges and are used to define attracting forces for each edge.

In general, image based methods can be very efficient. The main challenge when applying image based algorithms to 3D are the inherent rasterization steps. Image based models tend to project the $3 \mathrm{D}$ spatial information to a 2D case as well as cut away the geometric precision by rasterization. These steps can often be incorporated into a system as such. Eventually, visibility and depth-occlusion correct perspective 3D views may not easily be supported within such a visualization system.

In contrast to previous graph bundling approaches, our method works with geometric objects within a 3D environment, taking into account visibility and depth-occlusion problems, such that LOD-based interactive graph visualization can fully be integrated in 3D viewing applications. Moreover, recent publications have shown that interactive exploration of large graph datasets is crucial for effective online analysis. See also e.g. [22, 17, 8, 14, 18, 23] for example work on the aspect of interactivity in graph visualization. Our work also focuses on interactive and adaptive LOD constrained path bundling and visualization.

\section{CONSTRAINED GRAPH BUNDLING 3.1 Input Graph and Reference Vector Map}

Our input data consists of a dense graph $\mathbf{G}=(L, C)$ with spatial locations $L$ and many point-to-point connections $C \subseteq L \times L$. Typical representatives of such dense graphs are shown in Figs. 8(a), 9(a) and 10(a). Additionally we have a reference vector map, or reference graph $\mathbf{R}=(V, E)$ which defines a (planar) network of edges $E$ defined between its vertices $V$. An example of such a reference network is shown in Figs. 12(a). Furthermore, we define a trail $P=\left(V^{\prime}, E^{\prime}\right)$ in the reference graph $\mathbf{R}$ as a path of consecutive edges $E^{\prime}=\left\{e_{0}^{\prime} \ldots e_{k-1}^{\prime}\right\} \subseteq E$ connecting vertices $V^{\prime}=\left\{v_{0}^{\prime}, \ldots v_{k}^{\prime}\right\} \subseteq V$ with $e_{i}^{\prime}=\left(v_{i}^{\prime}, v_{i+1}^{\prime}\right)$. See also Fig. 3 for an example of input graph, reference network and trail paths.

\subsection{Bundling over Reference Graph}

The main goal of our approach is the combination of the above defined graph datasets $\mathbf{G}$ and $\mathbf{R}$ by bundling all the connections in G constrained to trajectory paths $P$ over the reference network $\mathbf{R}$. For this we first map the input locations $l_{j} \in L$ to their corresponding nodes $v_{j_{L}} \in V$ in the reference graph, and we find for each connection $c_{i} \in C$ a corresponding path $P_{i}$ in $\mathbf{R}$. Thus over the reference graph $\mathbf{R}$ we use a set of trails $\mathbf{T}=\left\{P_{i}\right\}$ implied by $C$ from the input graph $\mathbf{G}$.

By representing each graph connection $c_{i}$ by a path $P_{i}$ in $\mathbf{R}$ we achieve a constrained bundling since the general cluttered connections $C$ in $\mathbf{G}$ are now mapped onto a constrained set of path trails $\mathbf{T}$ over $\mathbf{R}$. The actual bundling in fact occurs due to the fact that multiple connections $c_{i_{1}}, \ldots i_{k}$ are eventually mapped onto trails $P_{i_{1}}, \ldots i_{k}$ which share sections of their paths in the reference network, see also Fig. 3(b).

For adaptive visual bundling and interactive visualization of the constrained bundling $\mathbf{T}$ we display all its paths $P_{i}$ as variable LOD

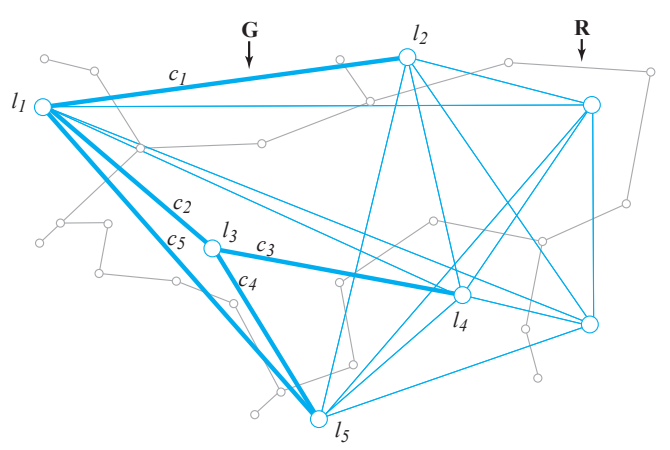

(a)

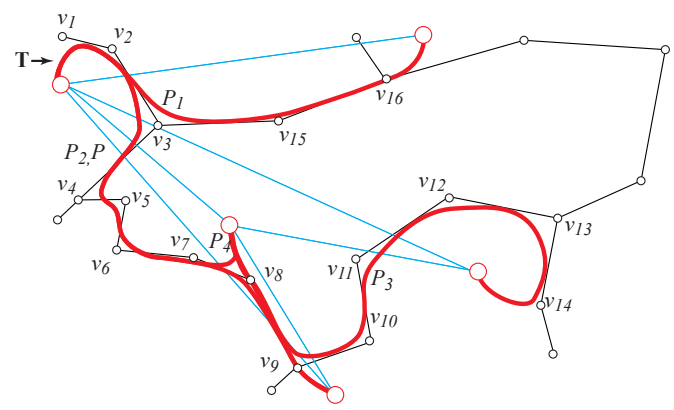

(b)

Figure 3: (a) Example input graph G (blue) over reference network $\mathbf{R}$ (grey) with a few point-to-point connections $c_{1 \ldots 5}$ highlighted. (b) Connections $c_{1 \ldots 5}$ bundled by B-spline curves, controlled by the vertices of the corresponding trails $\mathbf{T}=\left\{P_{1 \ldots 5}\right\}$ in the reference network $R$.

B-splines. The effect of the constraints and thus the visual outcome can be adjusted interactively at runtime, hence the bundling can be constrained more or less to the underlying reference network, and hence it could also be related to the accuracy of path $P_{i}$ representing the connection $c_{i}$ in the reference network $\mathbf{R}$.

Example vector map constrained bundlings are shown in Figs. 8(d), 9(b), 10(b) or 12(b) which use major roads, train rails or air corridors reference networks to bundle commuter, migration or flight data. In these examples, given the most likely point-to-point routings, the constrained bundling shows the potential impact of people movements or flights influencing the traffic on the different transportation network segments. With our method the transportation streams are constrained to and bundled along a reference network to follow realistic traffic paths routes, instead of bundling streams purely visually in $2 \mathrm{D}$ image or $3 \mathrm{D}$ object space.

Our algorithm consists of six preprocessing steps as indicated in Fig. 2. For performance reasons, the reference network $\mathbf{R}$ is first processed and simplified to a reduced graph $\widetilde{\mathbf{R}}$, minimizing the number of degree two nodes. A kd-tree index structure is then built that supports finding the nearest nodes in $\widetilde{\mathbf{R}}$ for all locations $L$ of G. Between these start- and endpoints, for each edge $c_{i}$ the shortest path $\widetilde{P}_{i}$ is computed in the reduced network $\widetilde{\mathbf{R}}$. Note that any other point-to-point routing according to the reference network semantic and properties could be used for this step to suit a particular application. Thereafter, a quadratic B-spline curve is defined with control points from the corresponding vertices of $P_{i}$ in the original network R. Based on additional obstacle avoidance constraints, 


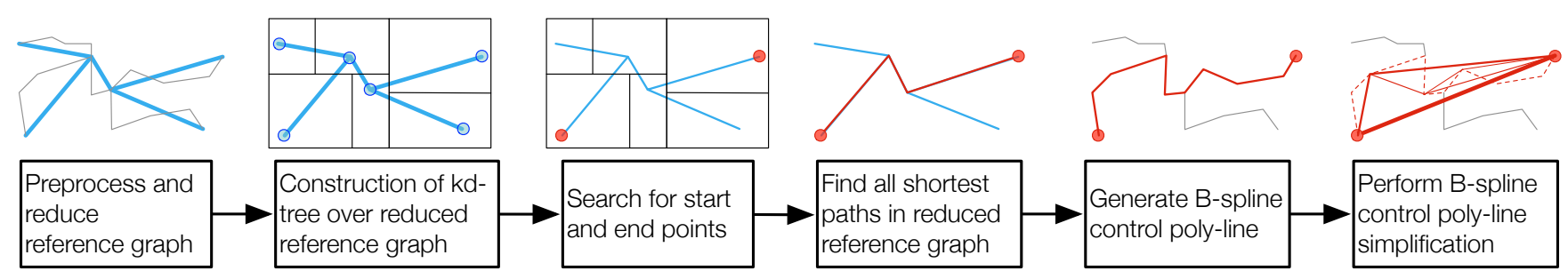

Figure 2: Overview of the preprocessing steps for our reference vector map constrained graph bundling.

hierarchical variable LOD B-splines are constructed using an iterative polyline simplification method.

\subsubsection{Reference graph reduction}

We first simplify the reference graph to cope with a large number of input locations $L$ and point-to-point connections $C$, so that costly point location and (shortest) path queries on detailed and complex reference networks $\mathbf{R}$ are avoided. The reduced undirected weighted reference graph $\widetilde{\mathbf{R}}$ is obtained by merging degreetwo nodes such that $\widetilde{\mathbf{R}}$ eventually only consists of endpoint and bifurcation nodes of $\mathbf{R}$ as illustrated in Fig. 4. Application specific edge weights and attributes, e.g. such as lengths, must be aggregated during these merging steps.

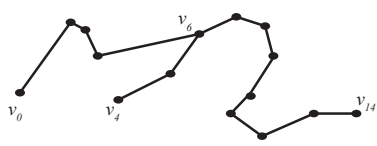

(a)

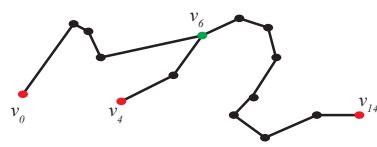

(c)

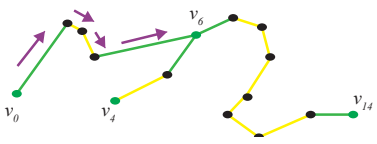

(b)

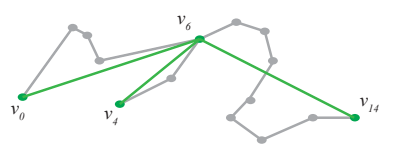

(d)
Figure 4: (a) Complete reference network R. (b) Traversal of reference network identifies endpoints and bifurcation points. (c) Endpoints (red) and bifurcation points (green) are retained, and degree-two points (black) can be removed. (d) Reduced reference graph $\widetilde{\mathbf{R}}$ shown in green.

The reference graph reduction is possible since for multiple trails $P_{i}$ sharing the same section $\left\{v_{j} \ldots v_{j+k}\right\}$ of a path between two bifurcation or endpoints $v_{j}$ and $v_{j+k}$, that section can be collapsed to a new reduced edge $\widetilde{e}_{j k}=\left(v_{j}, v_{j+k}\right)$. Note that shortest path queries over these collapsed edges $\widetilde{e}_{j k}$ are not affected as long as the path length of the sequence $\left\{v_{j} \ldots v_{j+k}\right\}$ is retained as aggregated edge weight for $\widetilde{e}_{j k}$.

Vector maps are often stored as a group of polylines from which the reference graph network $\mathbf{R}$ must first be built. This involves identifying all polyline endpoints and where they connect to other polyline vertices. In Figs. 4(b) and 4(c) 3 polylines $\left\{v_{0} \ldots v_{6}\right\}$, $\left\{v_{4} \ldots v_{6}\right\}$ and $\left\{v_{6} \ldots v_{14}\right\}$ are merged and the endpoints and crossings are marked. The reduced graph $\widetilde{\mathbf{R}}$ is then formed by omitting the polylines' degree-two vertices in between the marked nodes as in Fig. 4(d).

\subsubsection{Nearest neighbor and shortest path queries}

The next steps consist of mapping all locations $L$ of $G$ to their nearest points in $\widetilde{\mathbf{R}}$ and finding the corresponding paths for all connec- tions $C$. Instead of the shortest path finding described here, more specific application dependent routing rules could be applied for path finding in $\widetilde{\mathbf{R}}$. Using a kd-tree over the vertices of $\widetilde{\mathbf{R}}$ we can quickly find the vertices $\widetilde{v}_{i_{\text {start }}}, \widetilde{v}_{i_{\text {end }}} \in \widetilde{\mathbf{R}}$ corresponding to the endpoints $l_{i_{\text {start }}}, l_{i_{\text {end }}} \in L$ of each connection $c_{i} \in C$.

For each connection $c_{i}$ we are able to find the shortest path $\widetilde{P}_{i}=$ $\left\{\widetilde{\boldsymbol{p}}_{i_{\text {first }}}, \ldots \widetilde{\boldsymbol{p}}_{i_{\text {last }}}\right\}$ in $\widetilde{\mathbf{R}}$ between $\widetilde{v}_{i_{\text {start }}}$ and $\widetilde{v}_{i_{\text {end }}}$ using Dijkstra's shortest path algorithm. Many optimized and hardware accelerated methods exist to improve this step (see e.g. [7, 3]). Given all the paths $\widetilde{P}_{i}$ in the reduced graph $\widetilde{\mathbf{R}}$, the entire trails $\mathbf{T}=\left\{P_{i}\right\}$ in the complete reference network $\mathbf{R}$ can then easily be found by path expansion $\widetilde{P}_{i} \rightarrow P_{i}=\left\{\boldsymbol{p}_{i_{\mathrm{first}}}, \ldots \boldsymbol{p}_{i_{\text {last }}}\right\}$ to also include the leftout degree-two vertices.

These trails define polylines through $\boldsymbol{p}_{i_{\text {first }}}, \ldots \boldsymbol{p}_{i_{\text {last }}}$ which will serve as the control polygons for variable LOD B-spline curves as described below. Another advantage of our method, over other generic graph bundling approaches is, that until this point all information is in $3 \mathrm{D}$, i.e. the control points $\boldsymbol{p}_{i} \in \mathbb{R}^{3}$, and the B-spline interpolation and display can thus be done fully in $3 \mathrm{D}$.

\subsubsection{B-spline trail interpolation}

For visual bundling and display of trails $P_{i}=\left\{\boldsymbol{p}_{i_{\text {first }}}, \ldots \boldsymbol{p}_{i_{\text {last }}}\right\}$ we use endpoint interpolating quadratic B-spline curves $s_{i}(u)$. The control points $\boldsymbol{p}_{k}$ are given by the trail $P_{i}$ (with its point indices $i_{\text {first }} \ldots i_{\text {last }}$ remapped to $\left.k=0 \ldots K\right)$, and the quadratic B-spline of a trail path $P_{i}$ can then be written as:

$$
s_{i}(u)=\sum_{k=0}^{K} \boldsymbol{p}_{k} \cdot N_{k}^{2}(u)
$$

With the B-spline basis functions $N_{j}^{d}(u)$ given below, a quadratic B-splines $(d=2)$ can interpolate the endpoints $\boldsymbol{p}_{0}, \boldsymbol{p}_{K}$ by setting the knot values $u_{0}=u_{1}=u_{2}=0$ and $u_{K+1}=u_{K+2}=$ $u_{K+3}=1$, with the remaining $u_{j=3, \ldots K}$ of the knot vector $u_{j}$ being uniformly spaced.

$$
\begin{aligned}
& N_{j}^{d}(u)=\frac{u-u_{j}}{u_{j+d}-u_{j}} N_{j}^{d-1}(u)+\frac{u_{j+d+1}-u}{u_{j+d+1}-u_{j+1}} N_{j+1}^{d-1}(u) \\
& N_{j}^{0}(u)= \begin{cases}1 & u \in\left[u_{j}, u_{j+1}\right] \\
0 & \text { otherwise }\end{cases}
\end{aligned}
$$

Due to partition of unity property and the fact that $N_{j}^{d}(u)=0$ $\forall u \notin\left[u_{j}, u_{j+d+1}\right]$, degree- $d$ B-splines curve points $s_{i}(u)$ with $u \in\left[u_{j}, u_{j+1}\right]$ are strictly contained within the convex hull of the $d+1$ control points $\boldsymbol{p}_{j-d}, \ldots \boldsymbol{p}_{j}$. This property is important for our $3 \mathrm{D}$ intersection constrained variable bundling as outlined below, and for quadratic B-splines it means that a curve point for $u \in$ 


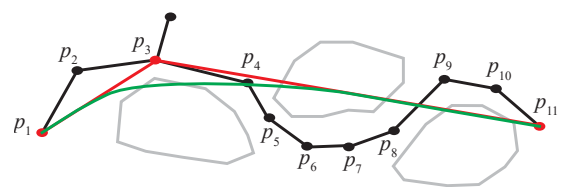

(a)

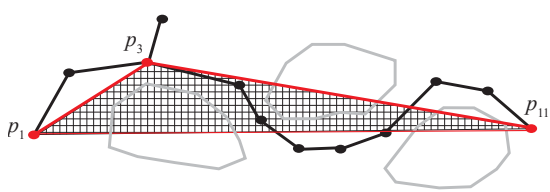

(b)

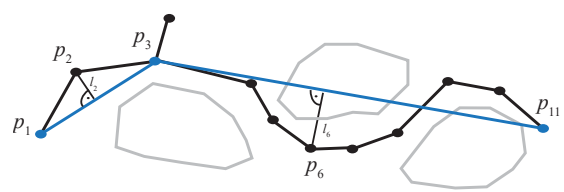

(c)

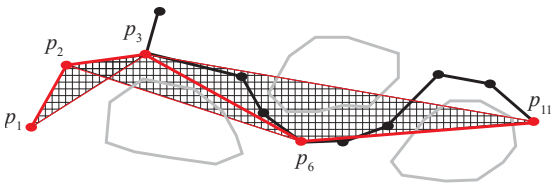

(d)

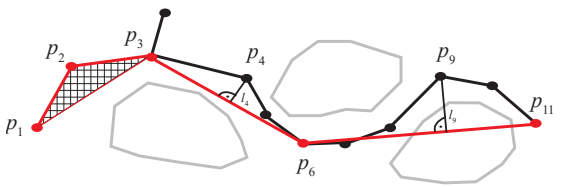

(e)

Figure 5: Example for a constrained quadratic B-spline control polygon refinement around obstacles. (a) Original B-spline (green) with minimal control polygon (red). (b) Intersection test of minimal control point triangle. (c) Douglas-Peucker refinement of the affected two line segments. (d) Recursive test of refined triangles for obstacle intersection. (e) Termination for one and recursive refinement of two other line segments.

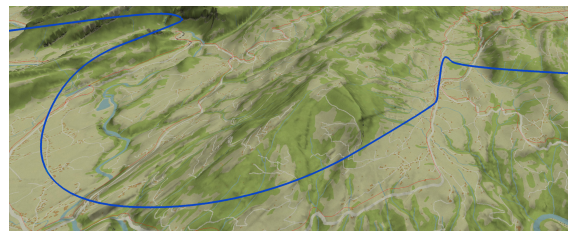

(a)

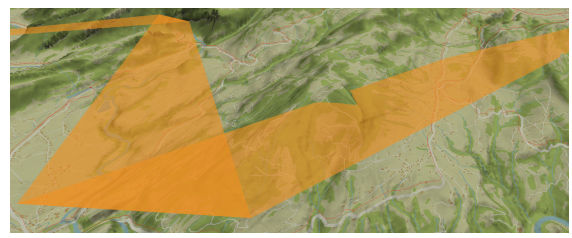

(d)

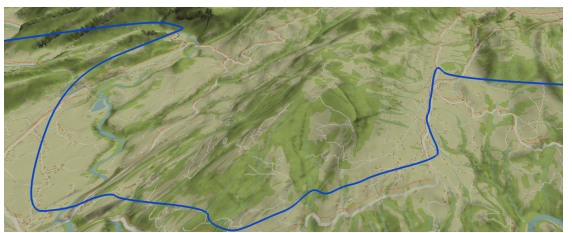

(b)

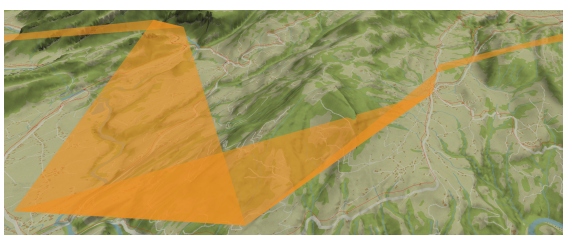

(e)

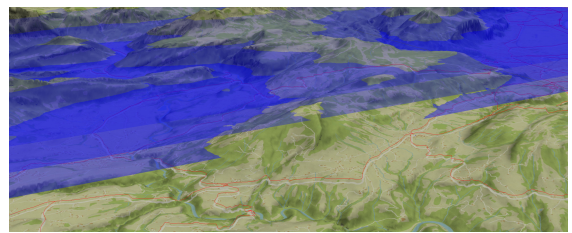

(c)

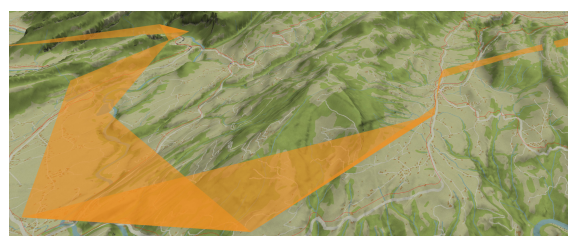

(f)

Figure 6: The 3D B-spline refinement levels from a perspective view. (a) Coarsest B-spline LOD, at high altitude offset, induced by reduced reference network graph. (b) Refined B-spline LOD at lower height offset avoiding terrain intersections. (c) Different height offsets (blue planes) at which 3D obstacle interferences are tested with respect to the terrain elevation model. (d,e,f) Quadratic B-spline refinements and control-point triangle occlusion queries evaluated against the 3D terrain at different height offsets.

$\left[u_{j}, u_{j+1}\right]$ is guaranteed to lie within the triangle $\triangle_{j-1}$ defined by the points $\boldsymbol{p}_{j-2}, \boldsymbol{p}_{j-1}$ and $\boldsymbol{p}_{j}$.

\subsubsection{Constrained B-spline simplification}

In a 3D environment, the bundled trail paths $P_{i}$ displayed as Bspline curves as described above may interfere with other 3D objects and obstacles. In particular, stream bundles following transportation network lines may intersect with the terrain height-field in a 3D geographic visualization system (see e.g. Figs. 6 and 11). In the following we describe how $3 \mathrm{D}$ obstacles can be taken into account to generate non-interfering quadratic B-splines, and more specifically, how we can define variable LOD B-splines around terrain height-field constraints in $3 \mathrm{D}$.

Occlusion queries in 3D scenes can effectively be implemented using graphics hardware [21]. For our approach we use hardware occlusion queries to define constrained B-splines through iterative polyline refinements using the Douglas-Peucker algorithm [20].

The principle of the Douglas-Peucker algorithm is to reduce a polyline through a point sequence $\left\{\boldsymbol{p}_{0}, \ldots \boldsymbol{p}_{K}\right\}$ initially to the line seg- ment $\overline{\boldsymbol{p}_{0}, \boldsymbol{p}_{K}}$. Then the next point $\boldsymbol{p}_{m}$ with $0<m<K$ is added which has the largest distance to the line $\overline{\boldsymbol{p}_{0}, \boldsymbol{p}_{K}}$. The resulting polyline $\left\{\boldsymbol{p}_{0}, \boldsymbol{p}_{m}, \boldsymbol{p}_{K}\right\}$ is then recursively processed in the same way for the two line segments $\overline{\boldsymbol{p}_{0}, \boldsymbol{p}_{m}} \rightarrow\left\{\boldsymbol{p}_{0}, \boldsymbol{p}_{l}, \boldsymbol{p}_{m}\right\}$ and $\overline{\boldsymbol{p}_{m}, \boldsymbol{p}_{K}} \rightarrow$ $\left\{\boldsymbol{p}_{m}, \boldsymbol{p}_{r}, \boldsymbol{p}_{K}\right\}$. This recursive refinement process defines a binary hierarchy over all the points $\boldsymbol{p}_{1}, \ldots \boldsymbol{p}_{l}, \ldots \boldsymbol{p}_{m} \ldots \boldsymbol{p}_{r} \ldots \boldsymbol{p}_{K-1}$ (see also Fig. 7), i.e. with root $\boldsymbol{p}_{m}$ with left and right child nodes $\boldsymbol{p}_{l}$ and $\boldsymbol{p}_{r}$ respectively.

As illustrated in Fig. 5, the key idea of our approach is to apply the Douglas-Peucker refinement principle on the control polygon of a trail's B-spline and use the convex hull property mentioned in Sec. 3.2.3 to define a sequence of point insertions. Hence for a trail $P_{i}=\left\{\boldsymbol{p}_{0}, \ldots \boldsymbol{p}_{K}\right\}$ we start with its initial minimal control points $\boldsymbol{p}_{j}^{0}=\widetilde{\boldsymbol{p}}_{i_{\text {first }}}, \ldots \widetilde{\boldsymbol{p}}_{i_{\text {last }}}$ (i.e. points $\boldsymbol{p}_{1}, \boldsymbol{p}_{3}, \boldsymbol{p}_{11}$ in Fig. 5(a)) implied by the reduced reference graph $\widetilde{\mathbf{R}}$. Starting with $l=0$, all triangles $\triangle_{j}^{l}=\left\{\boldsymbol{p}_{j-1}^{l}, \boldsymbol{p}_{j}^{l}, \boldsymbol{p}_{j+1}^{l}\right\}$ are tested for intersection with any 3D obstacle or other interference constraints (see Fig. 5(b)). If an intersection for $\triangle_{j}^{l}$ is detected, its two line segments $\overline{\boldsymbol{p}_{j-1}^{l}, \boldsymbol{p}_{j}^{l}}$ and $\overline{\boldsymbol{p}_{j}^{l}, \boldsymbol{p}_{j+1}^{l}}$ are subdivided by points from $\mathbf{R}$ (i.e. inserting points 


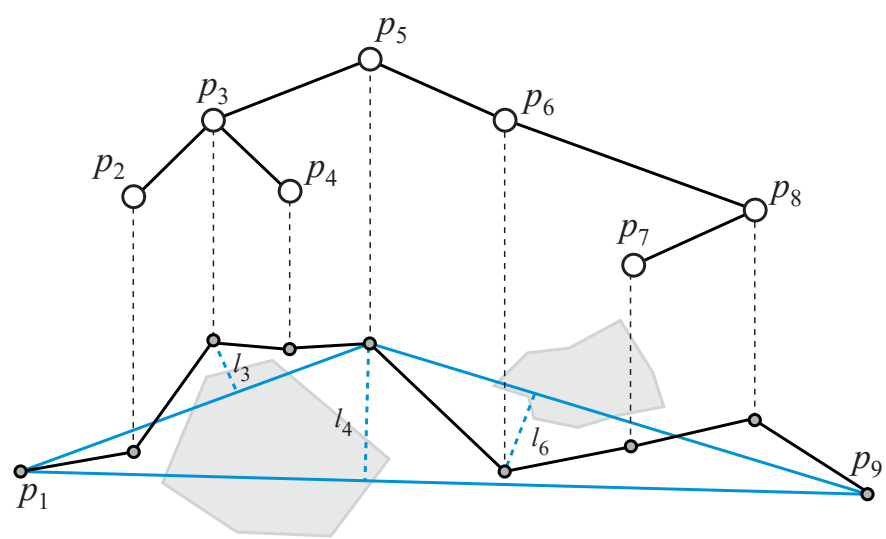

(a)

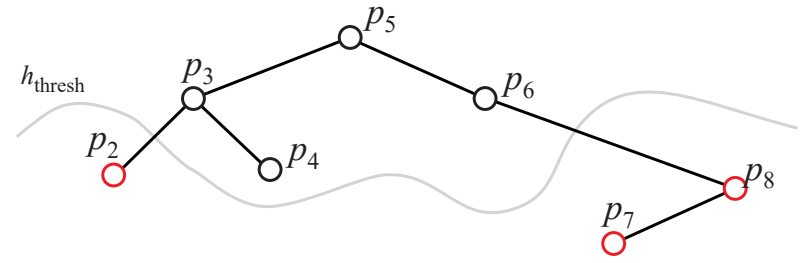

(b)

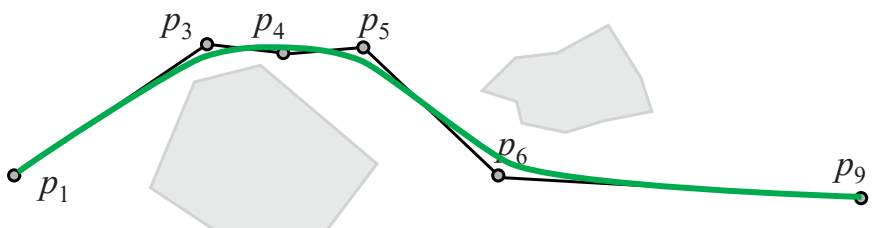

(c)

Figure 7: Variable LOD B-spline representation. (a) Original control polygon around obstacles and Douglas-Peucker induced binary vertex hierarchy. (b) Example control point selection using terrain intersection information (front elevation view). (c) Resulting control polygon selection guaranteeing a non-intersecting B-spline around obstacles (top-down view).

$\boldsymbol{p}_{2}$ and $\boldsymbol{p}_{6}$ in Fig. 5(c)) to form a refined set of control points $\boldsymbol{p}_{j}^{l+1} \in$ $\mathbf{R}$. This process is now recursively applied to the new triangles $\triangle_{j}^{l+1}$ (i.e. $\triangle_{2}, \triangle_{3}$ and $\triangle_{6}$ in Fig. 5(d)) until no more intersections are detected (e.g. $\triangle_{2}$ in Fig. $5(\mathrm{e})$ ).

\subsubsection{Variable LOD B-spline}

The variable LOD B-spline model is based on the Douglas-Peucker refinement and a binary hierarchy over consecutive segments of

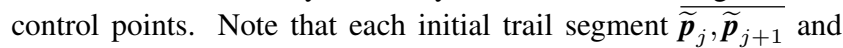
its subsequent Douglas-Peucker refinement as outlined above gives rise to a set of points added in between $\widetilde{\boldsymbol{p}}_{j}$ and $\widetilde{\boldsymbol{p}}_{j+1}$ which form a binary tree as illustrated in Fig. 7(a) which we refer to by $\widehat{\widetilde{\boldsymbol{p}}_{j} \widetilde{\boldsymbol{p}}_{j+1}}$. Hence each complex trail

$P_{i}=\left\{\widetilde{\boldsymbol{p}}_{0}, \boldsymbol{p}_{1}, \ldots, \boldsymbol{p}_{s-1}, \widetilde{\boldsymbol{p}}_{s}, \boldsymbol{p}_{s+1}, \ldots \boldsymbol{p}_{t-1}, \widetilde{\boldsymbol{p}}_{t}, \boldsymbol{p}_{t+1}, \ldots \boldsymbol{p}_{K-1}, \widetilde{\boldsymbol{p}}_{K}\right\}$

is now represented as a control polygon consisting of a sequence number of binary Douglas-Peucker refinement trees

$$
\widehat{P}_{i}=\widehat{\widetilde{\boldsymbol{p}}_{0} \widetilde{\boldsymbol{p}}_{s}}, \widehat{\widetilde{\boldsymbol{p}}_{s} \widetilde{\boldsymbol{p}}_{t}}, \ldots \widehat{\widetilde{\boldsymbol{p}}_{k} \widetilde{\boldsymbol{p}}_{K}},
$$

which together define a hierarchical LOD control polygon structure $\widehat{P}_{i}$ for the quadratic B-spline $s_{i}(u)$.
Given a certain LOD selection criterion that can be evaluated and stored in the control polygon structure $\widehat{P}_{i}$ and control point trees $\widehat{\boldsymbol{p}_{j} \widetilde{\boldsymbol{p}}_{j+1}}$, it is possible to variably adjust the control polygon and thus the detail level of the displayed B-spline $s_{i}(u)$ accordingly. At the lowest detail level, $s_{i}(u)$ is defined by the minimal control polygon points of $P_{i}$ only from $\widetilde{\mathbf{R}}$, i.e. only points $\widetilde{\boldsymbol{p}}_{0}, \widetilde{\boldsymbol{p}}_{s}, \widetilde{\boldsymbol{p}}_{t}, \ldots \widetilde{\boldsymbol{p}}_{K}$ in Eq. 2, while at the highest LOD the control polygon includes all points of $P_{i}$ from $\mathbf{R}$. Fig. 7(b) illustrates a geometry-occlusion based control point selection in the binary refinement tree giving rise to the control polygon in Fig. 7(c). The resulting B-spline will display a smooth curve following the reference network path $P_{i}$ but avoiding intersection with the given scene geometry.

Given a 3D digital terrain elevation model as B-spline simplification constraints, we apply the proposed framework as follows. For each trail $P_{i}$ given in a 3D terrain environment, we test the height field intersection constraints by issuing hardware accelerated occlusion queries for the control point triangles $\triangle_{j}^{l}$ on the $3 \mathrm{D}$ terrain obstacle at different elevations $h$ above ground as shown in Fig. 6. Starting with a maximal height offset $h_{\max }$ for $l=0$, we continuously reduce it for $l \rightarrow l+1$ (Fig. 6(c)) and record for every required control polygon refinement - adding new points $\boldsymbol{p}_{j \pm 1}^{l+1}$ because $\triangle_{j}^{l}$ interferes with the $3 \mathrm{D}$ terrain - the height offset at which the intersection occurs. Figs. 6(d) to $6(\mathrm{f})$ show the control point triangle intersection queries with the terrain at different height offsets. Thus for each trail segment tree $\widetilde{\boldsymbol{p}}_{s} \widetilde{\boldsymbol{p}}_{t}$, eventually all inner points $\boldsymbol{p}_{j}=\boldsymbol{p}_{s+1} \ldots \boldsymbol{p}_{t-1}$ record their height offset $h_{j}$ at which they must be included for B-spline refinement to avoid intersection with the 3D terrain environment. At rendering time, any variable LOD B-spline can thus be created by an inorder traversal of the tree segments $\widehat{\boldsymbol{p}}_{s} \widetilde{\boldsymbol{p}}_{t}$ of the control polygon hierarchy $\widehat{P}_{i}$, and by selecting all control points $\boldsymbol{p}_{j}$ for which their height offset $h_{j}$ is above a given threshold $h_{\text {thresh }}$. Variable LOD B-spline examples at different height offsets are rendering in Figs. 6(a), 6(b) and 11.

\section{INTERACTIVE CONSTRAINED GRAPH BUNDLE VISUALIZATION}

Given the input graph $\mathbf{G}$, reference vector map network $\mathbf{R}$ and a 3D digital elevation model, the preprocess as outlined in Sec. 3 and illustrated in Fig. 2 generates our proposed reference-network and terrain-height-field constrained variable LOD B-spline representations $\widehat{P}_{i}$ for all given point-to-point connections $c_{i}$.

At run-time, the visualization application's data preparation tasks are to select the B-splines control point trees $\widehat{P}_{i}$ of all paths $P_{i}$ to be displayed, and to generate the variable LOD B-spline control polygon from the points $\boldsymbol{p}_{j} \in \widehat{P}_{i}$ for which $h_{j}>h_{\text {tresh }}$ for a user given threshold $h_{\text {tresh }}$, i.e. height offset above ground. A drawable representation for each $\widehat{P}_{i}$ is then generated as an OpenGL 3D line-strip by evaluating $s_{i}(u)$ for small parameter intervals $\Delta u$. Alternatively, a De Boor based subdivision algorithm could be used to refine the control polygon such as to eventually use it as an OpenGL line strip primitive. Note that this processing step only has to be done once for each displayed trail when the height offset threshold $h_{\text {tresh }}$ changes.

Given all the B-splines to-be displayed as OpenGL 3D line strips, they can be rendered together with the 3D scene environment using standard 3D rendering techniques for surfaces, e.g. the grid digital terrain height field, and for line segments, i.e. the B-spline line strips. For a high quality result, our real-time rendering of the $3 \mathrm{D}$ 


\begin{tabular}{|c|c|c|c|c|c|c|c|c|c|c|c|}
\hline \multirow[t]{2}{*}{ Data sets } & \multicolumn{2}{|c|}{ G } & \multicolumn{2}{|c|}{$\mathbf{R}$} & Graph & \multicolumn{2}{|c|}{$\widetilde{\mathbf{R}}$} & \multirow{2}{*}{$\begin{array}{l}\text { Startpoint } \\
\text { queries }\end{array}$} & \multirow{2}{*}{$\begin{array}{l}\text { Shortest } \\
\text { path }\end{array}$} & \multirow{2}{*}{$\begin{array}{l}\text { Line } \\
\text { hierarchy }\end{array}$} & \multirow{2}{*}{ FDEB } \\
\hline & $|L|$ & $|C|$ & $|V|$ & $|E|$ & & $|\widetilde{V}|$ & $|\widetilde{E}|$ & & & & \\
\hline Vorarlberg: & 105 & 3442 & 9258 & 83017 & $0.1 \mathrm{~s}$ & 598 & 4586 & $0.1 \mathrm{~s}$ & $0.4 \mathrm{~s}$ & $4.5 \mathrm{~s}$ & $153 \mathrm{~s}$ \\
\hline d small. & 2886 & 17125 & 975491 & 902525 & $2.9 \mathrm{~s}$ & 36736 & 55033 & $1.8 \mathrm{~s}$ & $225.8 \mathrm{~s}$ & & $1-2 \mathrm{~h}$ \\
\hline Switzerland: & 2886 & 31608 & 975491 & 902525 & $2.9 \mathrm{~s}$ & 36736 & 55033 & $2.5 \mathrm{~s}$ & $424.5 \mathrm{~s}$ & $443.7 \mathrm{~s}$ & \\
\hline US Migration: & 3235 & 387841 & 65761 & 65177 & $0.01 \mathrm{~s}$ & 785 & 1072 & $8.7 \mathrm{~s}$ & $51.3 \mathrm{~s}$ & $102.3 \mathrm{~s}$ & - \\
\hline World Airlines: & 3457 & 68382 & 1778 & 1084 & $0.01 \mathrm{~s}$ & 395 & 798 & $4.4 \mathrm{~s}$ & $35.4 \mathrm{~s}$ & $6.6 \mathrm{~s}$ & \\
\hline
\end{tabular}

Table 1: Cluttered graph $(G)$ and reference network $(R)$ data sizes as well as processing times for reference graph reduction, nearest point shortest path queries, control point hierarchy generation and the force-directed edge bundling calculation. Due to memory limits, FDEB timings are available only for smaller graphs. Experiments were conducted on a system implemented in C++ and OpenGL using an Intel Core i7-3770K 3.50GHz, 16GB RAM, Geforce 680 GTX with 4GB RAM, and Windows 7.

terrain and B-spline line strips exploits a deferred shading pipeline using multiple framebuffer objects and stencil information.

The B-spline line-strip objects are rendered into a stencil-framebuffer such as to enable stencil based colorization and other more complex image processing operations. This allows image-based manipulations of the rendered B-spline curves before and together with the final compositing with the 3D scene environment. Currently the stencil is exploited for evaluating the bundling intensity by recording for each pixel how many B-splines contribute to it.

Besides the stencil based line strip rendering, all other 3D scene objects are rendered in one main pass into a separate buffer and combined with the line strip. A final composition stage merges all framebuffer information and delivers a super-sampled high resolution image for subsequent downsampling to the required screen resolution. We use a doubled resolution of all framebuffers to achieve a nice anti-aliased image in the end.

\section{EXPERIMENTAL RESULTS}

Our application allows to start and visualize the preprocessing phase for any combination of datasets. In particular, it supports exploration and visualization of the variable LOD reference-network bundled and 3D terrain-intersection constrained graphs in a full 3D environment at interactive frame rates. The current implementation is not optimized for performance but for the realization of the full functionality of the proposed constrained bundling framework.

Technical details as well as the computational cost of our reference bundling (REFB) can be found in Tab. 1. Our REFB method depends primarily on the input data complexity and has not yet been optimized for performance. Nevertheless, in comparison to FDEB (Fig. 8(b)) our approach scales better, primarily due to FDEB's quadratic memory and high computational cost requirements in the number of edges.

As shown in Fig. 8(d), the bundled commuter data makes it possible to identify which routes are used the most and which highways or main roads are most likely impacted from the commuter traffic. A comparison to FDEB in Fig. 8(b) shows that this can only be achieved if the bundling is constrained to some underlying and routing network information following hard constraints. The visual effect is similar to a street colorization as in Fig. 8(c). However the B-spline interpolation is smoother and shows more generalized paths which reduces the distraction for the user and emphasizes routing highlights better. The B-spline representation can also indicate the uncertainty of the applied (shortest path) routing in a visual sense. In comparison to a road colorization, the visual interpretation is not tied to an exact road. We argue that using a B- spline representation for transporting the information of uncertainty is preferable over a direct road colorization to identify highlights.

In addition to the comparison above, our method allows the integration of the bundled result in a three-dimensional scene and can take 3D graph and 3D vector map data into account but also supports 3D obstacle avoidance. As shown in Figs. 1, 6, 11, 12(c) and 12(d) bundling results can be displayed as variable LOD B-spline curves correctly in a $3 \mathrm{D}$ environment, thus supporting correct perspective viewing and depth-occlusions. The bundled variable LOD Bsplines can be rendered directly into a 3D scene environment and therefore integration of final image composition methods in a perspective view with complex depth-occlusions are possible.

In the most detailed LOD resolution the visual effect is similar to a street colorization. But for coarser detail levels, the adaptive Bspline LOD hierarchy can be exploited, and e.g. coupled with or used as indication of the uncertainty of the applied (shortest path) routing over the reference network. This allows to interactively change the geometry of path bundles based on an expected uncertainty value or other LOD attribute. If there is low evidence for commuter traffic following a specific route, path bundles can visualize this uncertainty in a geometric sense at lower LOD. This is based on the assumption that the geometric accuracy of the bundled paths following the constraint network routes improves the uncertainty information visually than using direct road colorization where this information does not appear.

\section{CONCLUSIONS AND FUTURE WORK}

In this paper we present a novel approach for vector-map constrained path bundling for 2D and 3D environments. Furthermore, we describe a hierarchical multiresolution control polygon structure that cannot only be used for rendering variable LOD B-splines, but which in particular can incorporate obstacle interference and intersection constraints in a 3D environment. Additionally we present a hardware occlusion-query based method for detecting intersections of (quadratic) B-spline segments with 3D obstacles.

The examples shown in the results are mainly from geospatial data sets, but our method is not domain specific to GIS systems. However, it requires and is targeted at a pair of co-registered (dense) input graph and reference network datasets embedded in a $2 \mathrm{D}$ or $3 \mathrm{D}$ environment. Our method is not aimed at general graphs or other simpler standard graphs which are sometimes used for testing in recent related work (e.g. as in [5, 12]). Nevertheless, bundling general graphs could be achieved by providing an artificial reference network or generating a reference network from the raw graph data itself, e.g. using the minimum spanning tree of the graph. In fact, an artificial reference network is shown in Fig. 10 for the world air- 


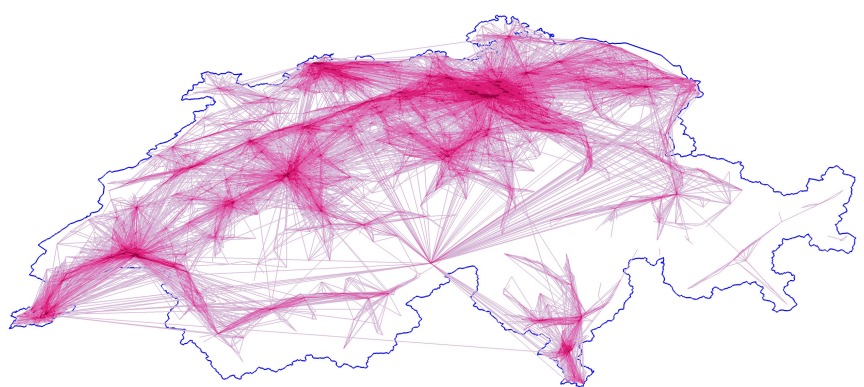

(a) Original data

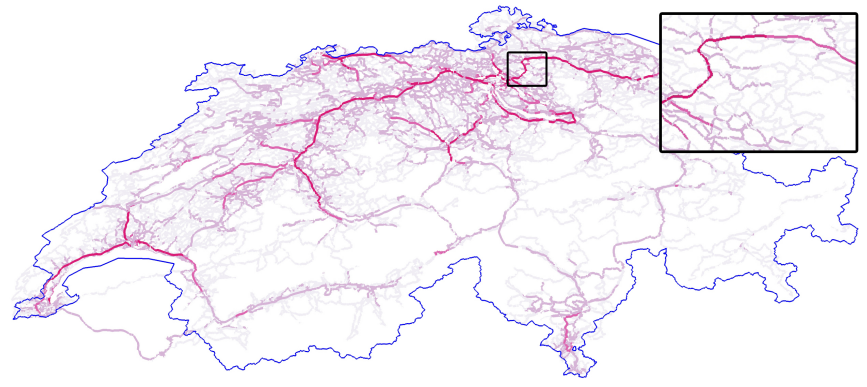

(c) Road Colorisation

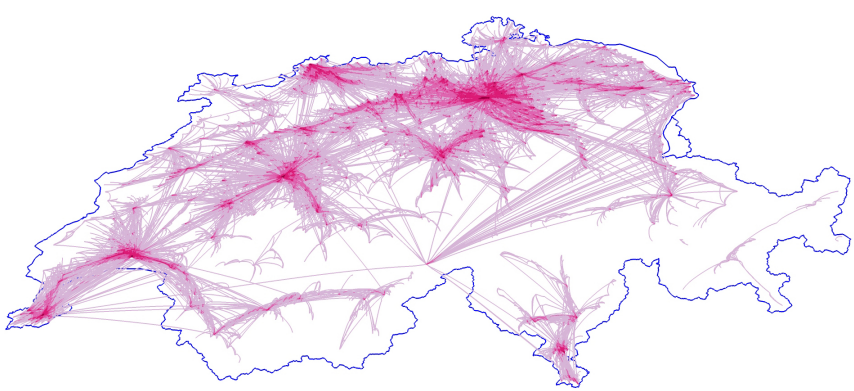

(b) FDEB

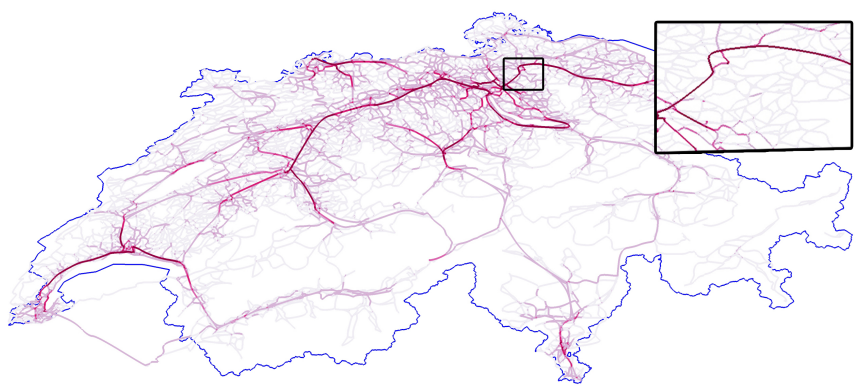

(d) REFB

Figure 8: Visual results of the Swiss commuter data. Strong colors indicate overlapping commuter streams. (a) Raw dataset containing the home-to-work travel information as town-to-town connections. (b) FDEB bundled graph. (c) Colored street data set. (d) Vector map constrained path bundling.

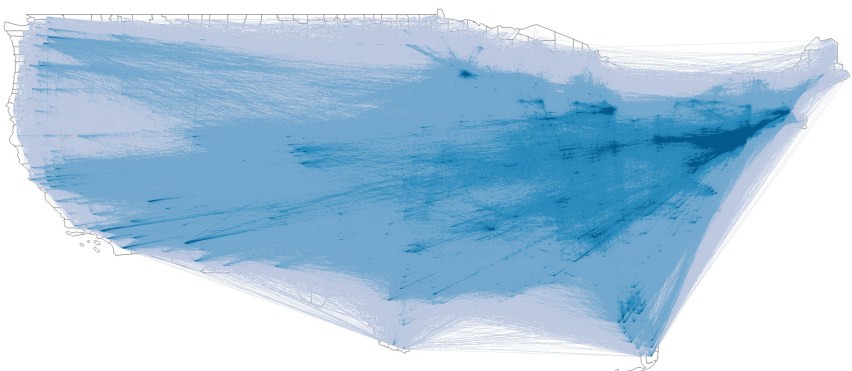

(a)

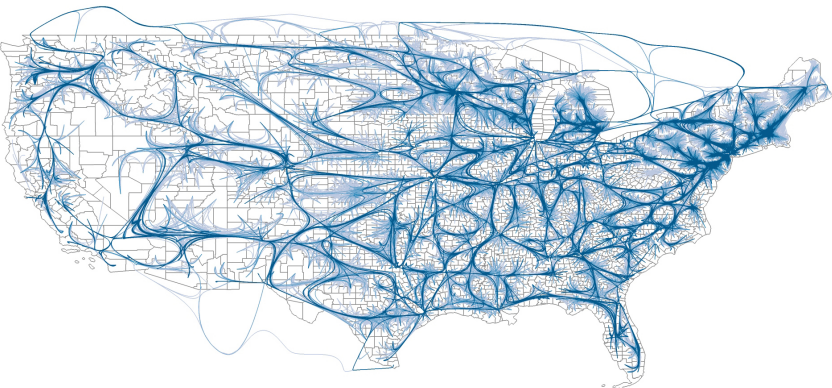

(b)

Figure 9: (a) The raw US migration dataset containing county-to-county migration information. (b) The bundled data set using the US train line data as reference network.

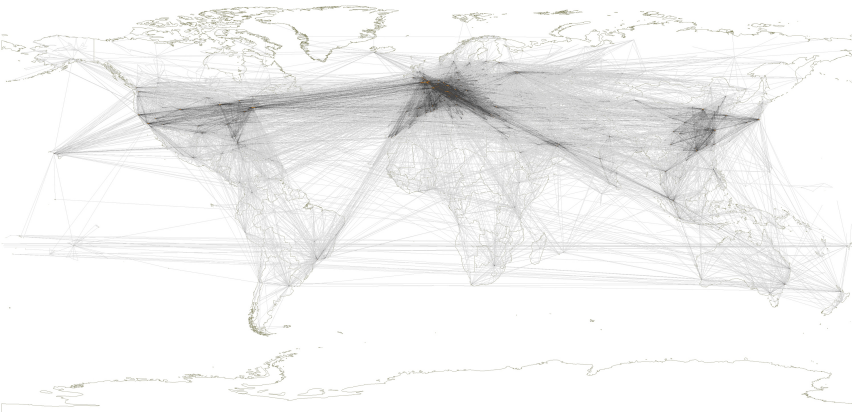

(a)

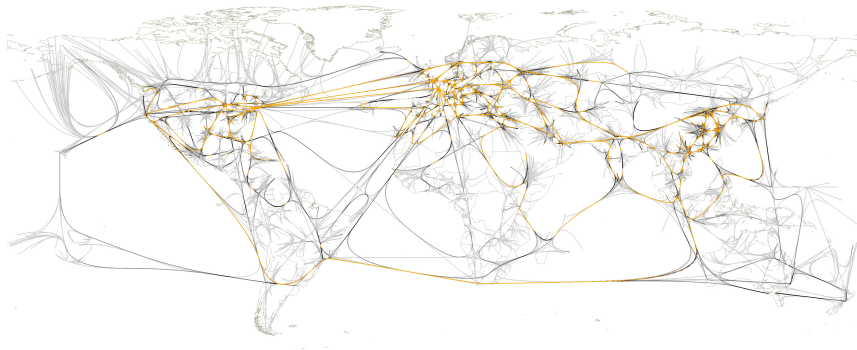

(b)

Figure 10: The world airline dataset (a) raw flight connections. (b) bundled over a virtual air corridor connection network with color encoded flight intensity (orange for heavier air traffic). 


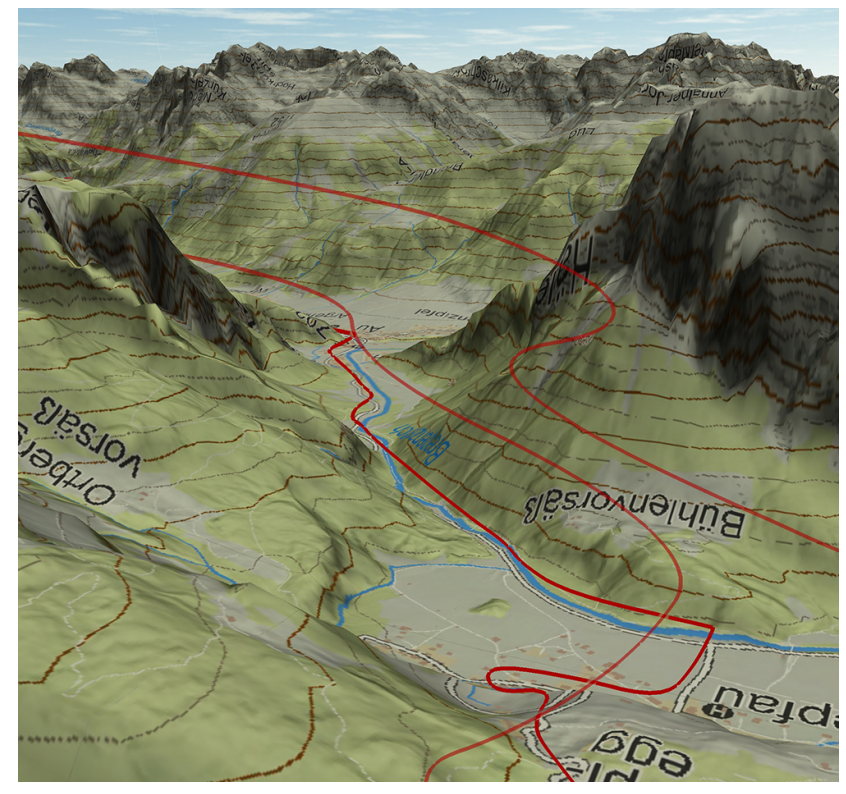

Figure 11: Screenshot showing different B-spline LODs at different height offsets over the 3D terrain.

line graph data, where the reference network of air corridors was modeled by cartographic domain experts according to their needs. In general, lots of reference networks exist in many domains, on top of which additional graph based relational information can be analyzed and visualized.

The major limitation of our method is the non-optimized implementation. For handling very large input graphs and reference networks of many 10 s or 100 s of thousands of connections, the variable LOD B-spline generation has to be improved in its use of hardware occlusion queries. Moreover, for large graphs the resulting massive amounts of line-strip geometry for the bundled B-splines cause limitations in interactivity. Thus only a subset of bundled B-splines can be explored interactively. These limitations are the main targets for future work. Further algorithmic improvements may be applicable to reference graph reduction, shortest path finding or routing, but these would primarily improve preprocessing but not the visual results. Furthermore, the precision of occlusion queries is limited. Occlusion queries depend on the viewport size and projection parameters, which may potentially lead to errors in the B-spline refinement procedure. Due to the similarity of this occlusion detection method to shadow maps, similar strategies could be applied such as multiple viewport snapshots to improve the data precision.

Thus the main limitations revolve around performance issues for handling massive vector map data sets. The performance of the variable LOD B-spline refinement has to be improved significantly as well as online line-strip generation and real-time rendering of massive amounts of line geometry.

\subsection{Streaming Applications}

In this paper we assume that the data sets are complete and available beforehand. This precludes potential use cases of path-bundling visualizations in a streaming environment. But the system we propose in this paper has the ability to be used in a data streaming environment as well. It is not necessary that the point-to-point data

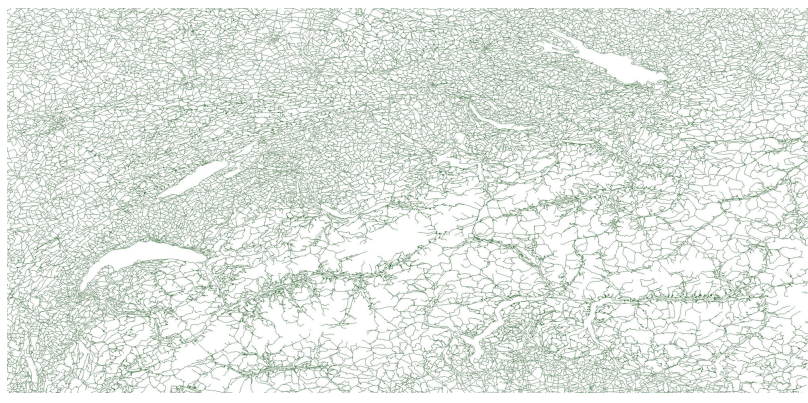

(a)

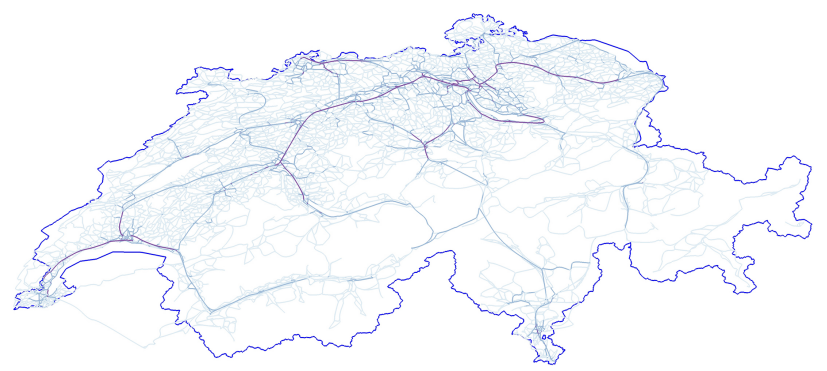

(b)

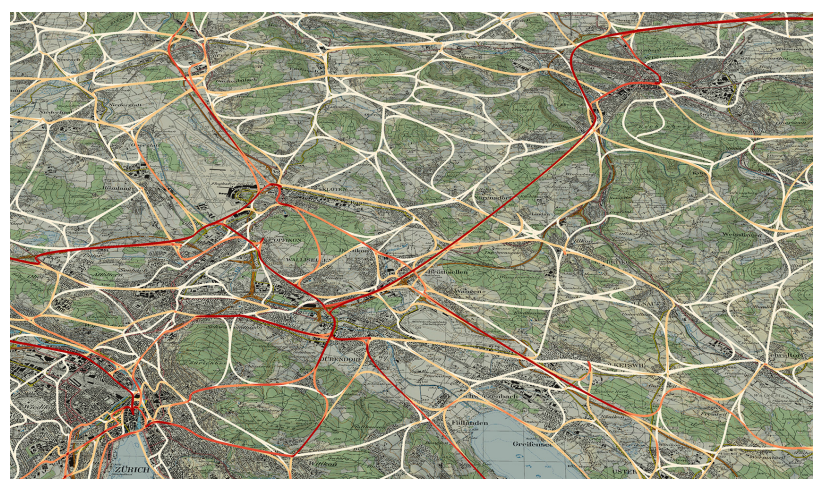

(c)

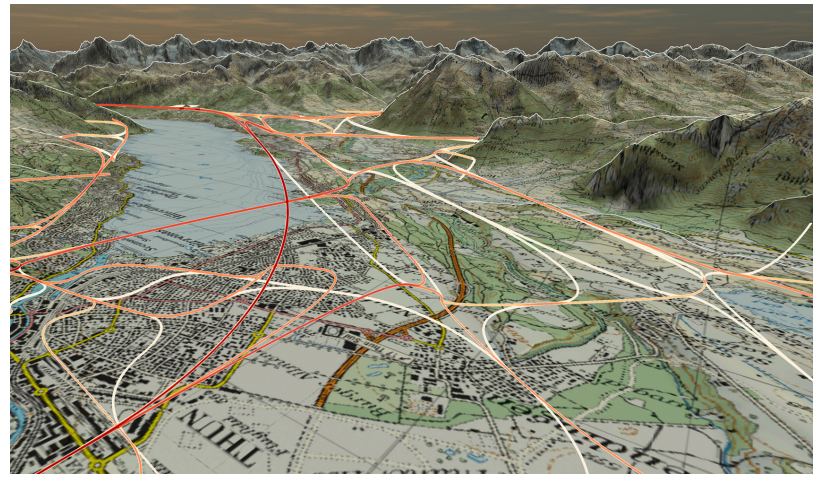

(d)

Figure 12: The results for Swiss commuter dataset in Fig. 8(a). (a) an example for a reference network showing major road data of Switzerland (1:200'000). (b) constrained bundling of commuter paths highlighting the major traffic axis visually as hot spots. (c) A zoom-in view of the highly frequented larger area of Zurich. (d) A perspective view snapshot including terrain context with edge highlights. 
sets are known and complete beforehand. Our approach offers the possibility to establish an on-demand service, for example for daily commuter travelers, daily taxi tours or other higher frequency connection data, where the connection stream is processed incrementally as connections are sequentially reported. This also means that one could maintain a time-window of actual connection data and bundled paths for visualization, drop old data and update it with newer connections based on the recorded time. By only taking a static reference network into account, a single path result will be consistent as if the method were applied on the entire data set at once, independent of how many bundles are processed and shown. This is a significant difference to approaches such as the FDEB method. The FDEB approach uses a precomputed measurement matrix storing relation weightings of all connections and calculates attraction forces between connections so that every point-to-point connection influences the final result. Therefore, in contrast to our method, the whole visualization can change with a single new connection and is thus not streaming-capable.

\section{ACKNOWLEDGMENTS}

The authors want to thank the Federal Office of Topography Swisstopo for providing the Swiss VECTOR25 and SwissTLM data sets as well as the Landesvermessungsamt Feldkirch, Austria, for providing the data sets of Vorarlberg.

\section{REFERENCES}

[1] P. Caserta, O. Zendra, and D. Bodenes. 3D hierarchical edge bundles to visualize relations in a software city metaphor. In Proceedings IEEE International Workshop on Visualizing Software for Understanding and Analysis, pages 1-8, 2011.

[2] W. Cui, H. Zhou, H. Qu, P. C. Wong, and X. Li. Geometry-based edge clustering for graph visualization. IEEE Transactions on Visualization and Computer Graphics, 14(6):1277 -1284, November/December 2008.

[3] D. Delling, A. V. Goldberg, A. Nowatzyk, and R. F. Werneck. PHAST: Hardware-accelerated shortest path trees. In Proceedings IEEE International Parallel \& Distributed Processing, pages 921-931, 2011.

[4] G. Ellis and A. Dix. A taxonomy of clutter reduction for information visualisation. IEEE Transactions on Visualization and Computer Graphics, 13(6):1216-1223, November/December 2007.

[5] O. Ersoy, C. Hurter, F. Paulovich, G. Cantareiro, and A. Telea. Skeleton-based edge bundling for graph visualization. IEEE Transactions on Visualization and Computer Graphics, 17(12):2364 -2373, December 2011.

[6] E. Gansner, Y. Hu, S. North, and C. Scheidegger. Multilevel agglomerative edge bundling for visualizing large graphs. In Proceedings IEEE Pacific Visualization Symposium, pages 187 -194, March 2011.

[7] A. V. Goldberg and C. Harrelson. Computing the shortest path: A search meets graph theory. In Proceedings ACM-SIAM Symposium on Discrete algorithms, pages 156-165, 2005.

[8] D. Guo. Flow mapping and multivariate visualization of large spatial interaction data. IEEE Transactions on Visualization and Computer Graphics, 15(6):1041 -1048, November/December 2009.

[9] D. Holten. Hierarchical edge bundles: Visualization of adjacency relations in hierarchical data. IEEE Transactions on Visualization and Computer Graphics, 12(5):741 -748, September/October 2006.
[10] D. Holten and J. J. van Wijk. Force-directed edge bundling for graph visualization. Computer Graphics Forum, 28(3):983-990, 2009.

[11] C. Hurter, O. Ersoy, S. I. Fabrikant, T. R. Klein, and A. C. Telea. Bundled visualization of dynamic graph and trail data. IEEE Transactions on Visualization and Computer Graphics, 99:1, 2013.

[12] C. Hurter, O. Ersoy, and A. Telea. Graph bundling by kernel density estimation. Computer Graphics Forum, 31:865-874, June 2012.

[13] C. Hurter, O. Ersoy, and A. Telea. Smooth bundling of large streaming and sequence graphs. In Proceedings IEEE Pacific Visualization Symposium, pages 41-48, 2013.

[14] A. Lambert, D. Auber, and G. Melançon. Living flows: Enhanced exploration of edge-bundled graphs based on gpu-intensive edge rendering. In International Conference Information Visualisation, pages 523 -530, 2010.

[15] A. Lambert, R. Bourqui, and D. Auber. 3D edge bundling for geographical data visualization. In International Conference Information Visualisation, pages 329-335, 2010.

[16] A. Lambert, R. Bourqui, and D. Auber. Winding roads: Routing edges into bundles. Computer Graphics Forum, 29(3):853-862, 2010.

[17] B. Lee, C. Plaisant, C. S. Parr, J.-D. Fekete, and N. Henry. Task taxonomy for graph visualization. In Proceedings AVI Workshop on Beyond Time and Errors: Novel Evaluation Methods for Information Visualization, pages 1-5, 2006.

[18] S.-J. Luo, C.-L. Liu, B.-Y. Chen, and K.-L. Ma. Ambiguity-free edge-bundling for interactive graph visualization. IEEE Transactions on Visualization and Computer Graphics, 18(5):810 -821, May 2012.

[19] D. Selassie, B. Heller, and J. Heer. Divided edge bundling for directional network data. IEEE Transactions on Visualization and Computer Graphics, 17(12):2354 - 2363, December 2011.

[20] W. Shi and C. Cheung. Performance evaluation of line simplification algorithms for vector generalization. The Cartographic Journal, 43:27-44, 2006.

[21] M. Wimmer and J. Bittner. Hardware occlusion queries made useful. In GPU Gems 2: Programming Techniques for High-Performance Graphics and General-Purpose Computation. Addison-Wesley, 2005.

[22] N. Wong and S. Carpendale. Interactive poster: Using edge plucking for interactive graph exploration. In Poster in the IEEE Symposium on Information Visualization, 2005.

[23] M. Zinsmaier, U. Brandes, O. Deussen, and H. Strobelt. Interactive level-of-detail rendering of large graphs. IEEE Transactions on Visualization and Computer Graphics, 18(12):2486 -2495, December 2012. 